\section{Le microbiote intestinal et la digestion des polysaccharides}

\author{
Abdessamad \&l Kaoutari ${ }^{1,3}$, Fabrice Armougom ${ }^{3}$, \\ Didier Raoult ${ }^{3}$, Bernard Henrissat ${ }^{1,2,4}$
}

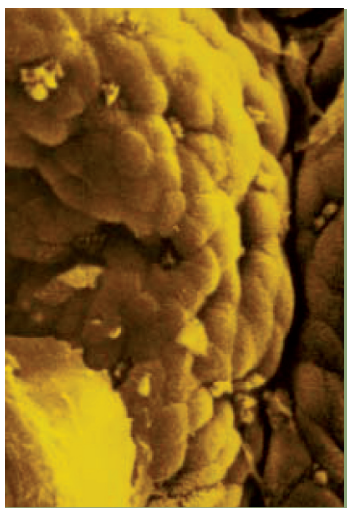

${ }^{1}$ Centre national de la recherche scientifique, UMR 7257, 13288 Marseille, France ; ${ }^{2}$ Université Aix-Marseille, architecture et fonction des macromolécules biologiques, 163, avenue de Luminy, 13288 Marseille Cedex 9, France ; ${ }^{3}$ URMITE, UM63, CNRS 7278, Institut de recherche pour le développement 198, Inserm 1095, université Aix-Marseille, faculté de médecine, Marseille, France;

méthodes de culture cellulaire. La difficulté à cultiver les bactéries anaérobies, qui composent majoritairement le microbiote intestinal, livrait cependant une vue trop partielle de sa biodiversité. Néanmoins, la dernière décennie a révélé l'immense biodiversité du microbiote intestinal

${ }^{4}$ Department of cellular and molecular medicine, faculty of health and medical sciences, University of Copenhagen, DK-2200 Copenhague, Danemark.

bernard.henrissat@ afmb.univ-mrs.fr et son impact potentiel sur la santé humaine, grâce au développement de technologies à haut débit couplées aux méthodes indépendantes de culture (métagénomique, pyroséquençage amplicon) [43].

Le microbiote intestinal distal correspond à l'ensemble des micro-organismes présents dans le côlon. C'est un écosystème complexe composé essentiellement de bactéries et, dans une moindre mesure, d'archées, d'eucaryotes et de virus. Le nombre de cellules bactériennes dans cet écosystème est estimé à $10^{14}$ et le nombre de gènes microbiens non redondants est, quant à lui, estimé à 3,3 millions [1], soit jusqu'à 150 fois plus que le nombre de gènes codant pour des protéines dans le génome humain (20 à 25 milliers) [2]. Cette importante population microbienne vit en symbiose avec son hôte et se nourrit de substrats végétaux et animaux issus de notre alimentation, ainsi que de substrats endogènes (mucines) propres à l'hôte.

Pendant longtemps, la caractérisation de la biodiversité du microbiote intestinal fut l'apanage des

Vignette (Photo (c) Inserm - Katy Haffen).

\section{Installation du microbiote}

Lors de la naissance, le mode d'accouchement détermine la primocolonisation bactérienne du tractus gastro-intestinal du nouveau-né, stérile à la naissance. Ainsi, l'enfant né par voie basse est d'abord colonisé par des bactéries d'origine maternelle, en particulier des bactéries vaginales, fécales ou cutanées [3]. Ensuite, les intestins du nouveau-né sont rapidement colonisés par les bactéries de l'environnement, essentiellement des Escherichia coli et des streptocoques, puis par celles contenues dans son alimentation, à base de lait, notamment les bifidobactéries et les lactobacilles. Plus tard, d'autres genres bactériens s'installent dans le tractus intestinal comme Bacteroides et Eubacterium. L'âge du sevrage est une étape transitoire qui induit de forts changements dans la composition du microbiote par l'introduction d'aliments solides et variés (légumes, féculents). Le microbiote intestinal d'un enfant, stabilisé après le sevrage, est considéré comme étant en équilibre entre les deuxième et troisième années [3]. 


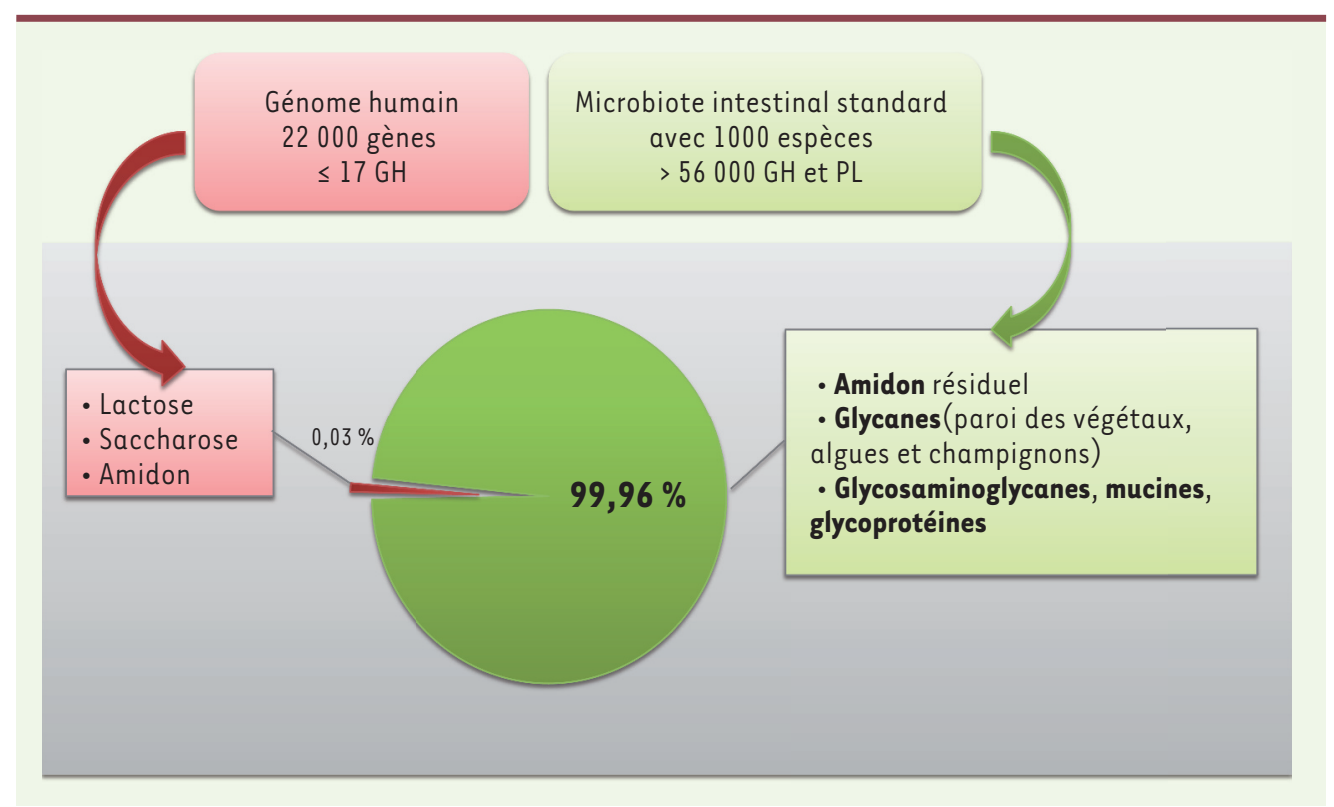

Figure 1. Potentiel digestif du microbiote intestinal humain. Le génome humain ne possède qu'au plus 17 gènes pour la digestion du lactose, du saccharose et de l'amidon. Le contraste est saisissant avec le microbiote intestinal dont environ 1000 espèces bactériennes codent pour plus de 56000 enzymes pour la digestion de l'ensemble des sucres complexes de notre alimentation, ainsi que ceux qui tapissent la surface des muqueuses intestinales. GH : glycoside-hydrolases; PL : polysaccharide-lyases.

\section{Diversité et composition}

La flore intestinale est un écosystème dynamique dont la composition peut varier considérablement au cours du temps [4], selon les états physiopathologiques et/ou les changements de régime alimentaire [5]. L'importante variabilité dans sa composition constatée entre les individus complique considérablement notre compréhension de la structuration et des fonctions du microbiote $[6,7]$. Par conséquent, l'identification d'un noyau phylogénétique composé des bactéries communes à différents individus (core microbiota) s'avère toujours difficile.

En revanche, la diversité du microbiote intestinal entre individus semble moins importante dès lors que l'on se place à un rang taxonomique supérieur comme le genre ou surtout le phylum (sur l'échelle de classification des espèces vivantes, le rang de phylum se place entre le règne et la classe). Les différentes analyses montrent que, parmi les 50 phylums bactériens connus, quatre sont prépondérants et communs dans le microbiote intestinal distal : (1) Firmicutes: principalement les genres Eubacterium, Clostridium, Ruminococcus, Butyrivibrio et Faecalibacterium; (2) Bacteroidetes: surtout Bacteroides, Prevotella et Porphyromonas; (3) Actinobacteria (Bifidobacteria); et (4) Proteobacteria (Escherichia). Malgré la présence quasi-universelle de ces principaux phylums dans le microbiote, leurs proportions varient considérablement d'un individu à un autre. L'étude d'une cohorte comprenant 242 volontaires américains sains, dans le cadre du projet de microbiome humain (human microbiome project), montre que la proportion des Firmicutes varie de $90 \%$ à moins de $10 \%$ selon l'individu, alors que la proportion des Bacteroidetes varie en sens inverse [7]. Ceci permet de relativiser les résultats d'études qui tendent à associer les maladies comme l'obésité à une réduction de la composition en Bacteroidetes du microbiote intestinal.
Enfin, étant donné que le microbiote est un système écologique complexe et clos, il semble que chaque relation microbe-hôte est caractérisée par une co-évolution continue orientée par les différents évènements au cours de la vie de l'hôte [8]. Le résultat de cette co-évolution est la mise en place d'un microbiote unique chez chaque individu.

Au contraire de l'importante biodiversité taxonomique, la diversité fonctionnelle du microbiote intestinal est moins importante entre les individus. Cette similitude des profils fonctionnels du microbiote a permis d'introduire le concept d'un noyau fonctionnel ou core microbiome au lieu d'un core microbiota (noyau phylogénétique) [7,9]. Ce noyau fonctionnel est constitué de familles de gènes retrouvées systématiquement avec une abondance similaire dans le microbiote d'individus différents, malgré des profils taxonomiques différents. La présence d'un noyau fonctionnel est accompagnée d'une stabilité fonctionnelle probablement due à la redondance fonctionnelle, où des dizaines, voire des centaines, de gènes de bactéries différentes coderaient pour la même fonction [10].

\section{Fonctions du microbiote intestinal}

Le rôle le plus important des bactéries intestinales est la digestion des substrats non digérés par l'hôte. Ce processus métabolique permet de fournir jusqu'à $10 \%$ des besoins énergétiques de l'homme [11]. D'autres rôles bénéfiques pour l'organisme ont été associés au microbiote intestinal, notamment la mise en place et la maturation du système immunitaire $[12,13,45](\rightarrow)$

$\rightarrow$ Voir la Nouvelle de N. Korneychuk, page 253 de ce numéro 
et la protection contre les micro-organismes pathogènes [14]. Dans cette revue, nous nous intéressons particulièrement aux fonctionnalités métaboliques du microbiote intestinal.

\section{Le microbiote intestinal et la digestion de l'indigestible}

La dégradation des sucres complexes présents dans nos aliments s'effectue au niveau du côlon par l'intermédiaire d'une grande variété d'enzymes appelées carbohydrate-active enzymes ou CAZymes [15]. La base de données CAZy (http://www.cazy.org/) fournit une classification des CAZymes en familles [16], et cette classification couvre notamment les glycoside-hydrolases (GH) et les polysaccharide-lyases (PL), deux classes de CAZymes qui catalysent la coupure des polysaccharides. Les CAZymes d'une même famille sont en général caractérisées par une machinerie catalytique identique, un mécanisme moléculaire similaire, mais des spécificités de substrats pouvant être différentes. Ces enzymes sont produites quasi-exclusivement par les bactéries intestinales. En effet, le génome humain ne code que pour 8 à $17 \mathrm{GH}$ qui présentent des capacités digestives limitées pour les sucres complexes (seuls sont digérés le lactose, le saccharose et l'amidon). En revanche, l'exploration de seulement 177 génomes bactériens de référence a permis d'identifier environ 10000 enzymes impliquées dans la digestion des sucres [17]. Le nombre de GH et PL qu'un microbiote de 1000 espèces peut produire est estimé à environ 56000 (Figure 1). Cet important arsenal enzymatique sert en premier lieu aux bactéries à assurer leur source de carbone en dégradant les substrats alimentaires non digérés.

\section{Principaux substrats indigestibles par l'homme et digérés par le microbiote}

Les fibres alimentaires sont des polysaccharides de plantes résistants à la digestion et à l'absorption au niveau de l'intestin grêle, mais qui subissent en revanche une fermentation complète ou partielle par le microbiote du côlon [18]. Les composants de la paroi de la cellule végétale et l'amidon non digéré par l'homme (amidon résiduel ou résistant) représentent les principales fibres alimentaires ciblées par les bactéries au niveau du côlon (Figure 2) [17].

\section{Amidon}

L'amidon est un polysaccharide complexe constitué de chaînes linéaires de molécules de glucose liées par des liaisons $\alpha-1,4$, et comportant des branchements $\alpha-1,6$ distribués de façon non aléatoire. Ce polysaccharide est présent dans les graines, les tubercules, les rhizomes et les fruits. Ce substrat est la cible des $\alpha$-amylases salivaire et pancréatique de l'homme, et l'absorption des produits d'hydrolyse est assurée au niveau de l'intestin grêle. La structure granulaire et insoluble de l'amidon cru limite cependant l'accès aux enzymes humaines, et sa dégradation est incomplète lorsque le bol alimentaire atteint le côlon [19]. Cette partie résiduelle de l'amidon est ciblée par le microbiote du côlon (Figure 2).
Composants de la paroi de la cellule végétale

La paroi de la cellule végétale est un élément structurel riche en polysaccharides complexes. Sa composition et sa structure peuvent différer d'une plante à l'autre en fournissant des substrats variés selon la plante consommée (fruits, légumes, céréales). La paroi végétale est composée de plusieurs constituants dont la cellulose, les hémicelluloses, les pectines, la lignine et les glycoprotéines. La cellulose est constituée de chaînes avec des centaines, voire des milliers, de résidus glucoses liés par des liaisons $\beta-1,4$. Les hémicelluloses servent de pontage entre les fibres cellulosiques, et peuvent contenir d'autres sucres que le glucose, comme le xylose, le mannose, le galactose ou encore le rhamnose. Quant aux pectines, ce sont des polysaccharides chargés, composés d'une chaîne principale d'acide uronique, au sein de laquelle s'intercalent des molécules de rhamnose, et de chaînes latérales à la composition en sucres extrêmement complexe.

\section{Glycosaminoglycanes et mucines}

Les glycosaminoglycanes sont des polysaccharides complexes d'origine animale et fongique, constitués de longues chaînes linéaires composées de répétitions de disaccharides contenant un sucre aminé et un acide uronique, comme l'acide glucuronique, ou un sucre neutre (tel que le galactose) [20]. Les glycosaminoglycanes sont apportés par l'alimentation d'origine animale puisqu'ils sont les composants majeurs de la matrice extracellulaire du tissu animal.

Certaines bactéries de la flore intestinale peuvent également digérer des glycanes endogènes de l'hôte, tels que les mucines. Les mucines sont des protéines hautement glycosylées recouvrant les surfaces muqueuses, dont les intestins. Au niveau du côlon, ces glycoprotéines forment une barrière physicochimique en séparant l'épithélium intestinal de la lumière colique (Figure 2).

\section{Fermentation des produits d'hydrolyse des polysaccharides}

La diversité des substrats végétaux contenus dans les aliments est telle que les GH et PL d'une seule bactérie ne suffisent pas pour dégrader tous les types de substrats, bien que le génome de certaines bactéries puisse coder pour des centaines de GH et PL différentes. La dégradation de la totalité des composants de la paroi de la cellule végétale nécessite donc la contribution de plusieurs espèces bactériennes, possédant un grand nombre d'activités enzymatiques différentes et 


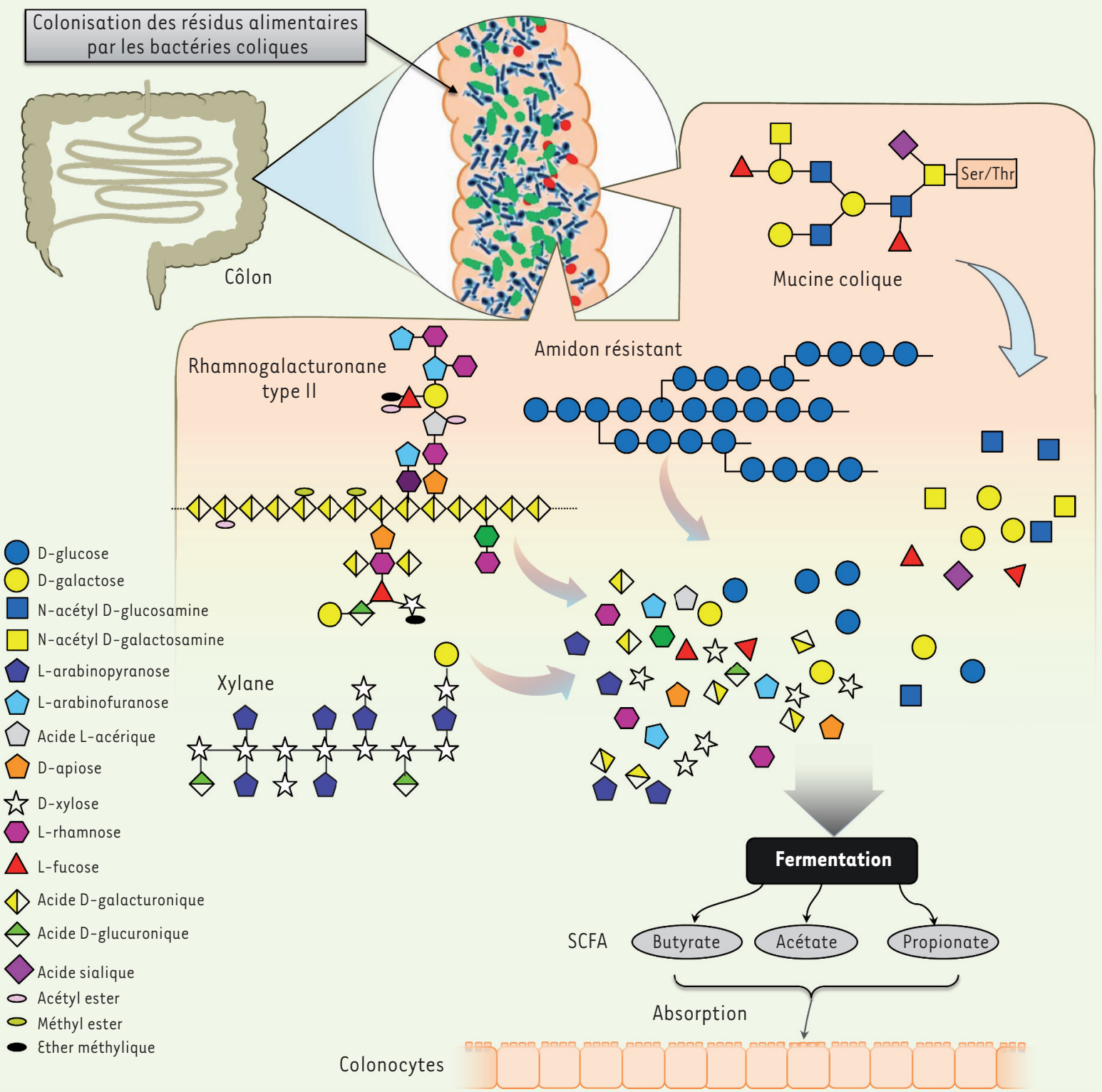

Figure 2. Digestion des sucres complexes par le microbiote intestinal. Hormis le lactose, le saccharose et la partie facilement digérable de l'amidon, I'homme est incapable de digérer les nombreux polysaccharides de son alimentation, dont la figure montre quelques exemples, comme le xylane, le rhamnogalacturonane de type II (un des constituants de la pectine) et la fraction d'amidon qui n'a pas été digérée dans l'intestin grêle. On appelle fibres ces polysaccharides non digérés, qu'ils aient une morphologie en forme de fibres ou pas. Par ailleurs, certaines bactéries de la flore intestinale sont capables de digérer les sucres complexes attachés aux protéines mucines qui tapissent la muqueuse intestinale. La digestion proprement dite consiste, d'abord, en une coupure par les enzymes bactériennes des polysaccharides en leurs sucres simples constitutifs, puis en la fermentation bactérienne de ces sucres simples en acides gras à courtes chaînes (acétate, propionate et butyrate) qui sont absorbés par nos colonocytes. SCFA : short chain fatty acid.

complémentaires [21]. Par exemple, la complexité d'un seul composant de la paroi de la cellule végétale, comme le rhamnogalacturonane de type II, nécessite l'intervention d'au moins 30 enzymes différentes pour son hydrolyse complète (Figure 2) [21].

Pendant le processus biochimique de digestion anaérobique, les chaînes polysaccharidiques sont coupées en chaînes plus courtes, puis en sucres simples, puis fermentées en métabolites intermédiaires comme l'acide formique, l'acide succinique et l'acide lactique. Les produits finaux de cette chaîne trophique sont les acides gras volatils ou SCFA (short-chain fatty acid), comme le butyrate, l'acétate et le propionate (Figure 2). 


\section{Les SCFA}

Le butyrate est le SCFA majeur issu de la fermentation des polysaccharides par le microbiote intestinal. Ce produit constitue la première source d'énergie pour les cellules du côlon (colonocytes), et son manque provoque l'autophagie ou l'autolyse de ces dernières qui finissent par se dégrader [22]. On attribue aux SCFA une multitude de fonctions bénéfiques, telles que l'inhibition de la prolifération des cellules tumorales du côlon et la stimulation de la croissance de colonocytes sains [23]. Les SCFA auraient également des propriétés antiinflammatoires via l'inhibition de l'activation du facteur nucléaire kappaB impliqué dans la réponse immunitaire [24], et ils peuvent jouer un rôle contre la résistance à l'insuline et contre l'obésité chez les souris, en augmentant les dépenses énergétiques [25].

\section{Microbiote et maladies}

\section{Obésité et modèles animaux}

La plupart des études métagénomiques du microbiote intestinal humain distal consistent à séquencer des fragments d'ADN bactérien extrait des selles. On distingue deux types d'études métagénomiques: (1) celles qui se focalisent exclusivement sur l'ADN codant pour l'ARN 16S des ribosomes et donnent la répartition taxonomique de la population bactérienne; et (2) celles qui séquencent un grand nombre de fragments d'ADN bactérien afin d'obtenir un échantillonnage au hasard des fonctionnalités codées par la flore microbienne. Les premières analyses métagénomiques du microbiote intestinal distal ont suggéré que l'obésité, chez l'homme et dans le modèle souris, serait liée à la diminution des Bacteroidetes résultant en un rapport Firmicutes/Bacteroidetes élevé [26, 44]. Cependant, cette corrélation n’a pas été confirmée ultérieurement. Bien au contraire, d'une part ce rapport ne changerait pas de manière significative avec la prise de poids [27] et, d'autre part, le changement de ce rapport se ferait en faveur des Bacteroidetes et non pas des Firmicutes [28].

L'analyse du microbiote fécal de souris rendues obèses par une mutation du gène codant pour la leptine (hormone qui contrôle la sensation de faim) a montré que l'abondance de la bactérie Akkermania muciniphila (appartenant au phylum Verrucomicrobia) est réduite de 3300 fois par rapport aux souris contrôles non mutées. En outre, le traitement par gavage oral de ces souris obèses avec un probiotique contenant $A$. muciniphila entraîne une forte réduction de poids et de la proportion de gras corporel [29]. Le mécanisme d'action d'A. muciniphila reste à éclaircir, mais il semble que cette bactérie, qui est capable de se nourrir des mucines de l'hôte, joue un rôle dans le maintien de la fonction de la barrière intestinale et module le taux d'absorption des nutriments (l'épaisseur de la couche de mucus intestinal est plus réduite chez les souris obèses) [29] $(\rightarrow)$.

$\rightarrow$ Voir la Nouvelle de P. Cani et A. Everard, $m / s n^{\circ} 2$, février 2014, page 125 Par ailleurs, le microbiote prélevé de souris obèses et introduit par gavage chez des souris dépourvues de flore intestinale induit une prise de poids significative et une capacité d'extraction ou de restitution calorique plus importante par rapport aux souris contrôles (souris sans germes transplantées avec une flore prélevée sur des souris normales) [30].

Afin d'étudier comment la flore intestinale réagit de façon dynamique à divers facteurs, on utilise un modèle animal faisant appel à des souris «humanisées », c'està-dire nées sans germes, puis ensemencées par gavage d'une flore prélevée chez des sujets humains. Ainsi, on a montré récemment que des souris humanisées avec une flore provenant de sujets obèses prenaient davantage de poids que celles ayant été ensemencées avec la flore d'individus maigres [31]. Les souris étant coprophages, on a placé dans la même cage les deux types de souris humanisées et, après quelques jours, on a constaté que la flore des souris maigres remplaçait peu à peu la flore des souris obèses. Cette meilleure performance de la flore des souris maigres est attribuée à une plus grande biodiversité de la flore «maigre » et à une meilleure adaptation à certains régimes alimentaires [31]. Ainsi, l'effet anti-obésité dominant de la flore «maigre» a été observé dans le cas d'un régime riche en polysaccharides végétaux, mais pas dans le cas d'un régime riche en graisses [31].

\section{Maladie de Crohn}

La maladie de Crohn est une maladie inflammatoire intestinale récemment attribuée à une altération du microbiote intestinal. Le microbiote des patients est caractérisé par une forte réduction des membres du phylum Firmicutes, en particulier Clostridium leptum [32] et Faecalibacterium prausnitzii, aussi bien en biodiversité qu'en abondance [33]. Cependant, cette corrélation négative entre $F$. prausnitzii et la maladie de Crohn a été remise en question dans d'autres études de cohortes de patients atteints de la maladie de Crohn qui suggèrent, au contraire, une augmentation significative de l'abondance de cette bactérie par rapport aux sujets sains [34]. De plus, l'amélioration clinique de ces patients s'accompagnerait d'une réduction de l'abondance de $F$. prausnitzii [35]. Ces résultats contradictoires montrent que les interactions microbiote-maladies sont plus complexes que la simple diminution ou augmentation de l'abondance d'une espèce bactérienne donnée ou d'un groupe bactérien particulier.

\section{Autres maladies}

Le déséquilibre du microbiote intestinal pourrait également être impliqué dans d'autres maladies inflammatoires, comme la rectocolite hémorragique [36], le syndrome de l'intestin irritable [36] et aussi dans des maladies auto-immunes telles que l'allergie [37], et dans les troubles du spectre autistique [38]. La liste des maladies pour lesquelles on implique le microbiote 
intestinal s'allonge pratiquement tous les jours [44] sans qu'un véritable lien de causalité entre le déséquilibre du microbiote et ces maladies ne soit réellement démontré. De plus, il faut considérer que l'altération de la composition du microbiote intestinal n'est peut être pas toujours la cause, mais parfois le résultat, des changements physiologiques accompagnant les pathologies en question.

\section{Prébiotiques, probiotiques et le contrôle de la flore intestinale}

Les sucres complexes non digérés par l'homme constituent la source majeure de carbone des bactéries de la flore intestinale. La quantité et le type de glucides apportés dans l'alimentation peuvent donc influencer considérablement les proportions entre les différentes bactéries intestinales et les fonctionnalités métaboliques associées [5, 15]. Des glucides spécifiques, appelés prébiotiques, sont utilisés pour tenter de manipuler le microbiote intestinal. La consommation d'un prébiotique particulier, comme l'inuline ou les fructo-oligosaccharides, favorise de façon transitoire la croissance des groupes bactériens Bifidobacteria, Faecalibacteria [39] et Roseburia [40]. Cependant, pour assurer une durabilité des effets bénéfiques des prébiotiques, il est nécessaire d'en consommer de façon régulière.

Les probiotiques sont des micro-organismes vivants ajoutés dans certains produits alimentaires, tels que les yaourts, dans le but d'excercer un effet positif sur la santé de l'hôte. Une étude récente utilisant des souris « humanisées » à l'aide d'un modèle de microbiote humain simplifié composé de 15 espèces bactériennes, a montré que le traitement avec des probiotiques (Bifidobacterium animalis, Lactobacillus delbrueckii, Lactococcus lactis et Streptococcus thermophilus) stimulait de façon significative l'expression d'un ensemble de gènes bactériens et, en particulier, le métabolisme de digestion des polysaccharides végétaux [41]. Ces résultats ont permis de conclure à un effet des probiotiques, mais cette conclusion est pour l'instant limitée aux souris « humanisées », car ces effets n'ont pas pu être mesurés chez l'homme [41]. Le véritable impact des probiotiques sur le microbiote intestinal humain reste donc à établir.

Une façon radicale d'induire un changement de composition du microbiote intestinal consiste à transplanter chez un patient la totalité du microbiote d'un sujet sain via une injection directe dans le côlon [42]. Contrairement à l'utilisation des probiotiques, qui ne sont composés essentiellement que de bactéries lactiques, le recours au transfert de l'ensemble des communautés d'un microbiote équilibré pourrait modifier et enrichir durablement un microbiote perturbé. Le rôle bénéfique de cette thérapie par transfert de microbiote intestinal a ainsi été démontré chez des patients atteints de colite ulcéreuse (ou rectocolite hémorragique) où l'on a observé une disparition complète des symptômes chez la totalité des patients ayant reçu un transfert de microbiote provenant de donneurs sains [42].

\section{Conclusions et perspectives}

Chaque microbiote intestinal individuel est un écosystème complexe dont les composantes microbiennes sont en équilibre dynamique. Les polysaccharides non digérés par l'homme fournissent le carbone nécessaire à la survie de la flore intestinale. Cette digestion par les enzymes bactériennes est essentiellement au bénéfice de la flore intestinale, puisque l'hôte récolte moins de $10 \%$ des calories issues de la digestion bactérienne [11]. À ce jour, les liens de causalité entre composition/structure microbienne de la flore intestinale et pathologies sont souvent suggérés, mais rarement vérifiés, les études aboutissant souvent à des résultats différents voire contradictoires [26-28]. Les fonctions métaboliques importantes, notamment celles nécessaires à la survie d'un tel écosystème, sont très souvent redondantes et présentes dans des groupes bactériens différents. Cette redondance procure au microbiote une grande résilience fonctionnelle, puisque la diminution ou la perte d'une espèce, voire d'un groupe phylogénétique entier n'entraîne pas la disparition des fonctions primordiales de l'écosystème. Cette résilience fonctionnelle est peut-être à l'origine des résultats contradictoires rapportés plus haut sur l'implication de groupes taxonomiques dans certaines pathologies, telles que l'obésité. En effet, il est parfaitement possible que, selon les individus, des microbiotes de composition taxonomique très différente possèdent des fonctionnalités similaires, et que des microbiotes de composition taxonomique similaire aient des profils fonctionnels différents.

Grâce aux nouvelles technologies de séquençage de I'ADN, notre compréhension de la complexité du microbiote digestif et de ses implications dans la santé humaine fait des progrès considérables. Cependant, la plupart des études se concentrent sur l'exploration de la diversité phylogénétique du microbiote, et peu appréhendent les aspects fonctionnels. L'accès aux régions plus centrales du tractus gastro-intestinal nécessitant des méthodes invasives relativement lourdes, les études du microbiote intestinal humain se sont essentiellement focalisées sur l'analyse de selles permettant un échantillonnage au niveau du côlon. En revanche, peu d'études explorent le microbiote de l'intestin grêle, notamment au niveau du jujénum ou de l'iléon. $\diamond$

\section{SUMMARY}

Gut microbiota and digestion of polysaccharides

The distal gut microbiota corresponds to all the microorganisms, essentially bacteria, that reside commonly in the colon. The microbial population is characterized by a large taxonomical diversity, counting approximately a thousand distinct bacterial species for a single individual. The pace of investigations of this microbial system has greatly accelerated these last few years, fuelled by the advent 
of metagenomics techniques, which do not rely on bacterial cultivation, but utilize high throughput DNA sequencing. In just a few years studies of the intestinal microbiota have become fashionable, albeit with often contradictory results when attempting to correlate changes in microbial composition to diverse pathologies. The article focusses on one of the essential functions of the distal gut microbiota: the digestion of the immense variety of polysaccharides from our diet that enzymes of the host cannot breakdown. $\diamond$

\section{LIENS D'INTÉRÊT}

Les auteurs déclarent n'avoir aucun lien d'intérêt concernant les données publiées dans cet article.

\section{RéFÉRENCES}

1. Qin J, Li R, Raes J, et al. A human gut microbial gene catalogue established by metagenomic sequencing. Nature $2010 ; 464: 59-65$.

2. Consortium IHGS. Finishing the euchromatic sequence of the human genome. Nature $2004 ; 431$ : 931-45.

3. Gronlund MM, Lehtonen OP, Eerola $\varepsilon$, Kero P. Fecal microflora in healthy infants born by different methods of delivery: permanent changes in intestinal flora after cesarean delivery. J Pediatr Gastroenterol Nutr $1999 ; 28: 19-25$.

4. Costello $\varepsilon$ K, Lauber CL, Hamady M, et al. Bacterial community variation in human body habitats across space and time. Science 2009 ; $326: 1694-7$.

5. Koropatkin NM, Cameron EA, Martens EC. How glycan metabolism shapes the human gut microbiota. Nat Rev Microbiol $2012 ; 10: 323-35$.

6. Eckburg PB, Bik $\varepsilon M$, Bernstein $C N$, et al. Diversity of the human intestinal microbial flora. Science $2005 ; 308: 1635-8$.

7. Structure, function and diversity of the healthy human microbiome. Nature $2012 ; 486: 207-14$.

8. Ley RE, Peterson DA, Gordon JI. Ecological and evolutionary forces shaping microbial diversity in the human intestine. Cell $2006 ; 124: 837-48$.

9. Kurokawa K, Itoh T, Kuwahara T, et al. Comparative metagenomics revealed commonly enriched gene sets in human gut microbiomes. DNA Res $2007 ; 14: 169-81$.

10. Turnbaugh PJ, Gordon JI. The core gut microbiome, energy balance and obesity. J Physiol 2009 ; $587: 4153-8$.

11. McNeil NI. The contribution of the large intestine to energy supplies in man. Am J Clin Nutr 1984 ; $39: 338-42$.

12. Cerf-Bensussan N, Gaboriau-Routhiau V. The immune system and the gut microbiota: friends or foes? Nat Rev Immunol $2010 ; 10: 735-44$.

13. Conroy ME, Shi HN, Walker WA. The long-term health effects of neonatal microbial flora. Curr Opin Allergy Clin Immunol 2009 ; 9 : 197-201.

14. Guarner F, Malagelada JR. Gut flora in health and disease. Lancet $2003 ; 361: 512-9$.

15. Cantarel BL, Lombard V, Henrissat B. Complex carbohydrate utilization by the healthy human microbiome. PLoS One 2012 ; 7 : e28742.

16. Cantarel BL, Coutinho PM, Rancurel C, et al. The Carbohydrate-Active EnZymes database (CAZy): an expert resource for glycogenomics. Nucleic Acids Res 2009 ; 37 : D233-8.

17. El Kaoutari A, Armougom F, Gordon Jl, et al. The abundance and variety of carbohydrate-active enzymes in the human gut microbiota. Nat Rev Microbiol $2013 ; 11: 497-504$.

18. DeVries JW. On defining dietary fibre. Proc Nutr Soc $2003 ; 62: 37-43$.

19. Gallant DJ, Bouchet B, Buleon A, Perez S. Physical characteristics of starch granules and susceptibility to enzymatic degradation. Eur J Clin Nutr 1992 ; 46 Suppl 2 : S3-16.

20. Esko JD, Kimata K, Lindahl U. Proteoglycans and sulfated glycosaminoglycans, $2^{\text {nd }}$ ed. Cold Spring Harbor (NY) : Cold Spring Harbor Laboratory Press, 2009.

21. Martens $\varepsilon C$, Lowe $\varepsilon C$, Chiang $H$, et al. Recognition and degradation of plant cell wall polysaccharides by two human gut symbionts. PLoS Biol $2011 ; 9$ : e1001221.

22. Donohoe DR, Garge N, Zhang $X$, et al. The microbiome and butyrate regulate energy metabolism and autophagy in the mammalian colon. Cell Metab $2011 ; 13: 517-26$.

23. Vanhoutvin SA, Troost FJ, Hamer HM, et al. Butyrate-induced transcriptional changes in human colonic mucosa. PLoS One $2009 ; 4$ : e6759.
24. Inan MS, Rasoulpour RJ, Yin L, et al. The luminal short-chain fatty acid butyrate modulates NF-kappaB activity in a human colonic epithelial cell line. Gastroenterology $2000 ; 118: 724-34$.

25. Gao Z, Yin J, Zhang J, et al. Butyrate improves insulin sensitivity and increases energy expenditure in mice. Diabetes $2009 ; 58: 1509-17$.

26. Ley RE, Turnbaugh PJ, Klein S, Gordon JI. Microbial ecology: human gut microbes associated with obesity. Nature 2006 ; 444 : 1022-3.

27. Zupancic ML, Cantarel BL, Liu Z, et al. Analysis of the gut microbiota in the old order Amish and its relation to the metabolic syndrome. PLoS One 2012 ; $7:$ : 43052 .

28. Schwiertz A, Taras D, Schafer $K$, et al. Microbiota and SCFA in lean and overweight healthy subjects. Obesity (Silver Spring) $2010 ; 18: 190-5$.

29. Everard A, Belzer C, Geurts L, et al. Cross-talk between Akkermansia muciniphila and intestinal epithelium controls diet-induced obesity. Proc Natl Acad Sci USA 2013; 110 : 9066-71.

30. Turnbaugh PJ, Ley RE, Mahowald MA, et al. An obesity-associated gut microbiome with increased capacity for energy harvest. Nature 2006 ; 444 : 1027-31.

31. Ridaura VK, Faith JJ, Rey FE, et al. Gut microbiota from twins discordant for obesity modulate metabolism in mice. Science 2013 ; 341 : 1241214.

32. Manichanh C, Rigottier-Gois L, Bonnaud $\varepsilon$, et al. Reduced diversity of faecal microbiota in Crohn's disease revealed by a metagenomic approach. Gut $2006 ; 55: 205-11$.

33. Sokol H, Pigneur B, Watterlot L, et al. Faecalibacterium prausnitzii is an anti-inflammatory commensal bacterium identified by gut microbiota analysis of Crohn disease patients. Proc Natl Acad Sci USA 2008; 105 : 16731-6.

34. Hansen R, Russell RK, Reiff C, et al. Microbiota of de-novo pediatric IBD: increased Faecalibacterium prausnitzii and reduced bacterial diversity in Crohn's but not in ulcerative colitis. Am J Gastroenterol 2012 ; 107 : 1913-22.

35. Jia W, Whitehead RN, Griffiths L, et al. Is the abundance of Faecalibacterium prausnitzii relevant to Crohn's disease? FEMS Microbiol Lett 2010 ; 310 : 138-44.

36. Kassinen A, Krogius-Kurikka L, Makivuokko H, et al. The fecal microbiota of irritable bowel syndrome patients differs significantly from that of healthy subjects. Gastroenterology $2007 ; 133: 24-33$.

37. Sjogren $Y M$, Jenmalm MC, Bottcher MF, et al. Altered early infant gut microbiota in children developing allergy up to 5 years of age. Clin Exp Allergy $2009 ; 39: 518-26$.

38. Parracho HM, Bingham MO, Gibson GR, McCartney AL. Differences between the gut microflora of children with autistic spectrum disorders and that of healthy children.J Med Microbiol 2005 ; 54 : 987-91.

39. Meyer D, Stasse-Wolthuis M. The bifidogenic effect of inulin and oligofructose and its consequences for gut health. Eur J Clin Nutr 2009 ; 63 : 1277-89.

40. Scott KP, Martin JC, Chassard C, et al. Substrate-driven gene expression in Roseburia inulinivorans: importance of inducible enzymes in the utilization of inulin and starch. Proc Natl Acad Sci USA 2011 ; 108 (suppl 1) : 4672-9.

41. McNulty NP, Yatsunenko T, Hsiao A, et al. The impact of a consortium of fermented milk strains on the gut microbiome of gnotobiotic mice and monozygotic twins. Sci Transl Med $2011 ; 3$ : 106ra.

42. Borody TJ, Warren EF, Leis $S$, et al. Treatment of ulcerative colitis using fecal bacteriotherapy. J Clin Gastroenterol 2003 ; 37 : 42-7.

43. Bernardo $P$, Albina $\varepsilon$, Eloit $M$, Roumagnac P. Métagénomique virale et pathologie. Med Sci (Paris) 2013 ; $21: 501-8$.

44. Burcelin $\mathrm{R}$, Chabo C, Blasco-Baque $\mathrm{V}$, et al. Le microbiote intestinal à l'origine de nouvelles perspectives thérapeutiques pour les maladies métaboliques? Med Sci (Paris) 2013 ; $29: 800-6$

45. Korneychuk $N$. Les cellules lymphoïdes innées contrôlent la réponse adaptative aux bactéries commensales intestinales. Med Sci (Paris) 2014 ; $30: 253-7$

\section{TIRÉS À PART}

B. Henrissat

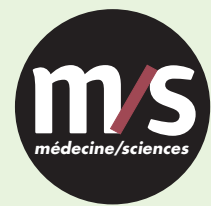

Tarifs d'abonnement $\mathrm{m} / \mathrm{s}-2014$

Abonnez-vous

à médecine/sciences
$>$ Grâce à $m / s$, vivez en direct les progrès des sciences biologiques et médicales

Bulletin d'abonnement page 334 dans ce numéro de $\mathrm{m} / \mathrm{s}$

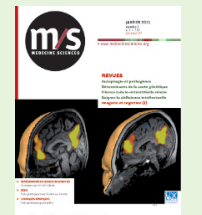

